\title{
Open Source EEG Platform with Reconfigurable Features for Multiple-scenarios
}

\author{
Juan M. López ${ }^{1}$, Fabián González ${ }^{2}$, Juan C. Bohórquez ${ }^{3}$, Jorge Bohórquez ${ }^{4}$, Mario A. Valderrama ${ }^{5}$, \\ Fredy Segura-Quijano ${ }^{6}$ \\ ${ }^{1,2,3,6}$ Departamento de Ingeniería Eléctrica y Electrónica, Universidad de los Andes, Colombia \\ ${ }^{4}$ Biomedical Engineering Department, University of Miami, United States of America \\ ${ }^{5}$ Departamento de Ingeniería Biomédica, Universidad de los Andes, Colombia
}

\begin{abstract}
Article Info
Article history:

Received Jun 28, 2018

Revised Sep 6, 2018

Accepted Sep 20, 2018

\section{Keyword:}

Biofeedback

Brain-Computer Interface

(BCI)

Electroencephalography (EEG)

Neurophysiology

Portable instruments

ABSTRACT

Electroencephalogram (EEG) acquisition systems are widely used as diagnostic and research tools. This document shows the implementation of a reconfigurable family of three affordable 8-channels, 24 bits of resolution, EEG acquisition systems intended for a wide variety of research purposes. The three devices offer a modular design and upgradability, permitting changes in the firmware and software. Due to the nature of the Analog FrontEnd (AFE) used, no high-pass analog filters were implemented, allowing the capture of very low frequency components. Two systems of the family, called "RF-Brain" and "Bluetooth-Brain", were designed to be light and wireless, planned for experimentation where movement of the subject cannot be restricted. The sample rate in these systems can be configured up to 2000 samples per second (SPS) for the RF-Brain and 250 SPS for the BluetoothBrain when the 8 channels are used. If fewer channels are required, the sampling frequency can be higher (up to $4 \mathrm{kSPS}$ or $2 \mathrm{kSPS}$ for 1 channel for RF-Brain and Bluetooth-Brain respectively). The third system, named "USBBrain", is a wired device designed for purposes requiring high sampling frequency acquisition and general purpose ports, with sampling rates up to 4 kSPS.
\end{abstract}

Copyright ()$^{2} 2018$ Institute of Advanced Engineering and Science. All rights reserved.

\section{Corresponding Author:}

Juan M. López,

Departamento de Ingeniería Eléctrica y Electrónica,

Universidad de los Andes,

Bogotá 111711, Phone: (+571) 3394949 ext 3375, Colombia

Email: jm.lopez580@uniandes.edu.co

\section{INTRODUCTION}

Flexible electroencephalogram (EEG) acquisition systems are required in a wide variety of research fields including brain computer interfaces (BCI) [1], [2], evoked potentials (EPs) [3], [4] stress response [5], [6], epilepsy [7]-[9], depth of anesthesia [10]-[12], among others. EEG systems' extended use is due to its noninvasive nature, high temporal resolution and lower cost compared with other modalities of brain activity monitoring such as functional magnetic resonance imaging (fMRI) or magnetoencephalography (MEG). Also, EEG devices do not require the restriction of the locomotion of the subject under test.

Commercial EEG research systems do not always offer flexibility to configure their settings, such as sampling frequency, location and configuration of the electrodes and individual gain of the channels, among others; and their prices can be high [13], [14]. The accessibility to these main features is critical in the experimentation design. Depending on the study, it could be more convenient to have less active channels and a higher sampling rate [15], [16]. Also, the selection of the reference electrode could significantly affect the quality of the acquired data [17]-[19], but changing and testing different locations is time consuming; thus, the possibility of selecting the reference electrode by software, among the electrodes already positioned 
in the scalp of the subject, would facilitate the assessment of the reference electrode location. The introduction of bias circuits in the EEG systems also improve the acquired signals by reducing the interference of the common-mode and motion artifacts [20]. The function of a bias circuit is equivalent to the right leg drive (RLD) circuits used in ECG systems. The possibility of selecting the source of the bias signal could result in a better EEG signal. The reconfigurable systems presented in this work provide a suitable experimentation tool at a lower cost than commercially available devices, for a broad range of electrophysiological studies. They offer the possibility of changing critical features including sampling rate, number of active channels, individual channel gain and channel input configuration to address the situations mentioned above.

Figure 1 presents a block diagram of the family called "the brain family". Three systems were implemented: RF-Brain, Bluetooth-Brain and USB-Brain. RF-Brain and Bluetooth-Brain are portable, battery-powered devices allowing experimentation where the movement of the subjects is not restricted; for this reason, the size of these systems was kept as small as possible. On the other hand, USB-Brain is powered by the USB port of the host computer where the signals are displayed and/or stored. All devices have a common core composed by the AFE and the control and configuration modules. A. Modules of the Bluetooth-Brain Transmitter can connect to an Android mobile device or to a PC with Bluetooth connectivity. B. Modules composing the USB-Brain, which must be connected directly to a USB port of the host PC. C. Modules for the RF-Brain Transmitter and RF-Brain Receiver. The RF-Brain Receiver connects to a PC through a USB port

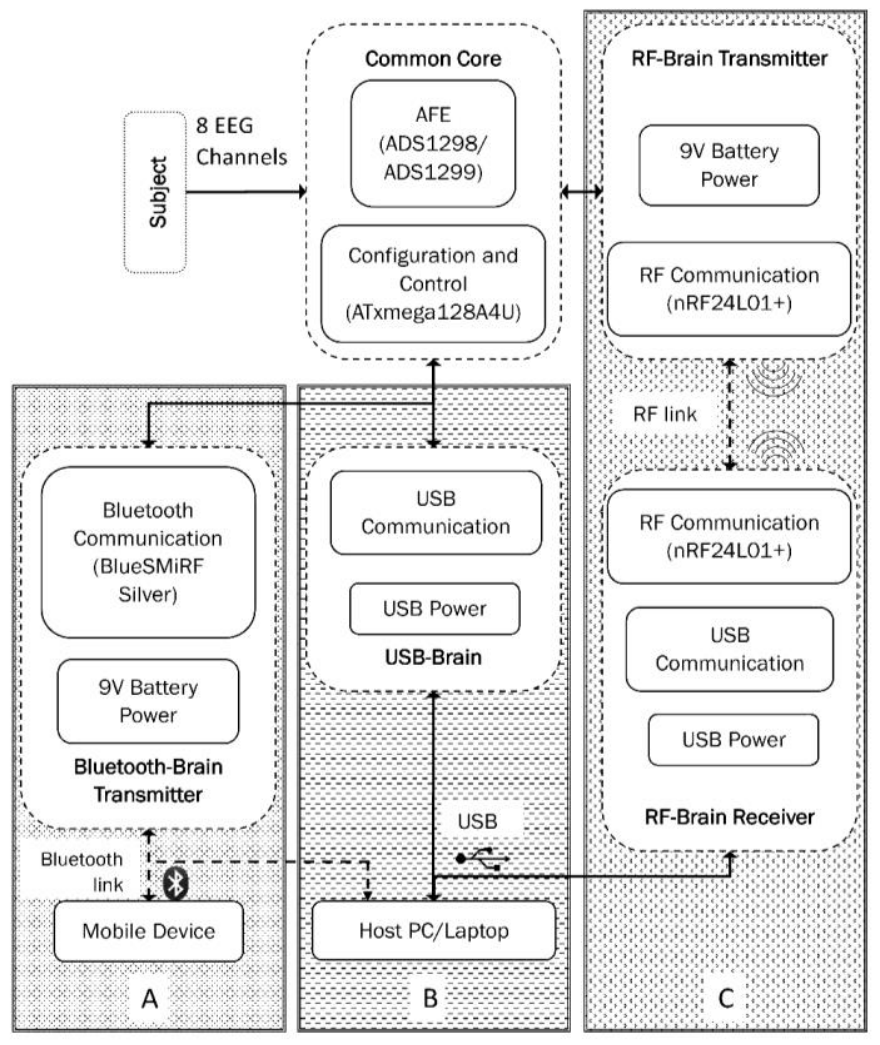

Figure 1. Block diagram of the Brain family of EEG acquisition systems

All the systems have three main components: hardware for acquisition, firmware that controls the behavior of the systems and a computer or mobile device software for configuration, display and storage of data, through a graphical user interface (GUI). These components were designed in a modular fashion in order to provide the required flexibility in their settings. Devices are open-hardware and open-software. Through a communications to the authors, researchers can retrieve all the necessary information to replicate the systems. 


\section{MATERIALS AND METHODS}

\subsection{Core of The Family}

All designed systems share a common core (see figure 1) composed by two modules: analog frontend (AFE) and configuration and control.

\subsubsection{AFE}

Depending on the system, the AFE module may use one of the following integrated circuits (ICs) from Texas Instruments ${ }^{\circ}$ : ADS1298 or ADS1299. Both ICs have eight channels with an instrumentation programmable gain amplifier (PGA) each one and 24 bit sigma-delta analog-to-digital converter (ADC). PGAs offer a set of possible gains from 1 to 24 . The resolution in bits of the ADC, along with these set of gains provide an approximately resolution of $12 \mathrm{pV}$. For 16 bit systems, the required gain to achieve the same resolution would have to be in the order of 103. AFEs maximum input bias current is $200 \mathrm{pA}$ and $300 \mathrm{pA}$ for the ADS1298 and the ADS1299 respectively. These ICs have an input multiplexer per channel for device noise measurement, test signal generation and acquisition, temperature measurement and supply voltage measurement. Main features of the two ICs are listed in Table 1.

Table 1. Features of The ICs Used in The AFE Modules ${ }^{\mathrm{a}}$

\begin{tabular}{lll}
\hline Feature & ADS1299 & ADS1298 \\
\hline Analog channels & 8 & 8 \\
Power consumption & $5 \mathrm{~mW}$ per channel & $0.78 \mathrm{~mW}$ per channel \\
Maximum input bias current & $300 \mathrm{pA}$ & $200 \mathrm{pA}$ \\
Voltage supply & Analog: $4.75 \mathrm{~V}$ to $5.25 \mathrm{~V}$ & Analog: $2.7 \mathrm{~V}$ to $5.25 \mathrm{~V}$ \\
& Digital: $1.8 \mathrm{~V}$ to $3.6 \mathrm{~V}$ & Digital: $1.65 \mathrm{~V}$ to $3.6 \mathrm{~V}$ \\
Sampling frequency & $250 \mathrm{SPS}$ to $16 \mathrm{kSPS}$ & 250 SPS to $32 \mathrm{kSPS}$ \\
CMRR & $-110 \mathrm{~dB}$ & $-115 \mathrm{~dB}$ \\
Programmable gain & $1,2,4,6,8,12$ or 24 & $1,2,3,4,6,8$ or 12 \\
Resolution in Volts & $268.22 \mathrm{pV}$ at gain=1 & $143.05 \mathrm{pV}$ at gain=1 \\
& $11.17 \mathrm{pV}$ at gain=24 & $11.92 \mathrm{pV}$ at gain=12 \\
Resolution in bits & 24 & 24 \\
Input-referred noise & $1.62 \mu \mathrm{Vpp}(70 \mathrm{~Hz}$ of band width at gain=6) & $4 \mu \mathrm{Vpp}(150 \mathrm{~Hz}$ of band width at gain=6) \\
\hline
\end{tabular}

The ADS1298 was used in the RF-Brain and Bluetooth-Brain as it offers a lower power consumption and a bigger supply voltage range as shown in Table 1, reducing the complexity of the energy module and therefore the space required in the printed circuit board (PCB). These features are desired for portable devices in order to offer more autonomy and less space and weight. Since the USB-Brain takes the energy from the USB port, the restriction in power consumption is not relevant, for that reason the ADS1299 was used, offering a better input-referred noise and a bigger gain (up to 24) as shown in Table 1. In all systems, the individual channel gain and the behavior of the input multiplexer can be selected from the user interface or modified by firmware. These settings are sent to the configuration and control module and are established at the beginning of the acquisition process.

In the Bluetooth-Brain system, all channels are single-ended, referred to a fixed electrode called reference electrode which also serves as bias electrode. The RF-Brain also has single-ended channels, but it offers the option of selecting the reference electrode between an independent electrode and one of the channel electrodes; these settings are selected through the GUI and an analog switch located on the board of the system, as shown in Figure 2. With the analog switch, the user can select to connect all negative inputs to the reference electrode or to the pin called SRB2, which can be selected among any electrode connected to the positive input of the AFE. The bias signal can also be configured with the help of a switch and the user interface. Figure 3 shows the internal and external diagram of this implementation. Bias signal may be formed from a set of electrodes selected by the user from the GUI and can be connected to the subject with the bias electrode. Its purpose is to reduce the common-mode interference by creating a negative feedback loop to the subject, equivalent to the RLD signal in ECG systems. If this is not required, the user can select the bias signal to be the mid-supply voltage. The external electronics explained above were designed based on the ADS1299EEG-FE performance demonstration kit, the information of which is available online in www.ti.com.

A. Internal diagram of the ADS 1299 or ADS1298. SRB2 can be connected to any individual positive channel input and SRB1 can be connected to all negative channel inputs. B. When using singleended inputs, through a switch the user can select if the reference is the reference electrode or one of the channel electrodes connected to SRB2. When operating in differential mode, SRB1 and SRB2 are not connected to any channel input. 


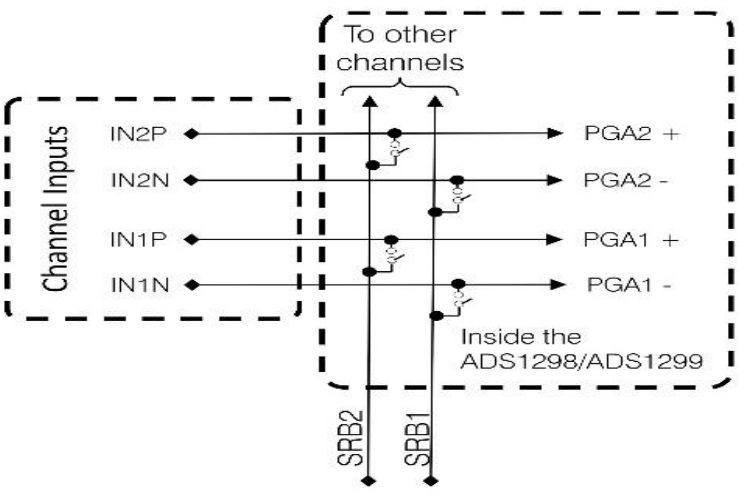

(a)

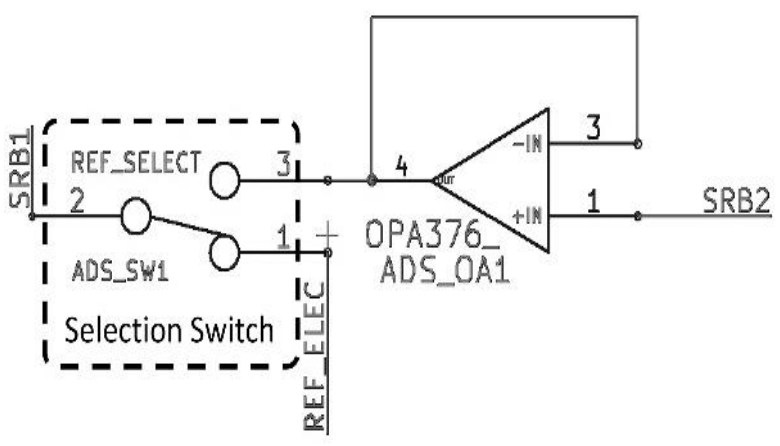

(b)

Figure 2. Circuit and internal diagram of the variable reference circuit

In the case of the USB-Brain system, the user can configure the channels to be differential or singleended. In the single-ended configuration, the reference and the bias signal can be set in the same way as for the RF-Brain (figures 2 and 3). No analog high-pass filters were implemented, allowing capture of signals from DC and avoiding possible distortions due to the nonlinearities of the analog filters. Systems were designed to be used with floating type, cup electrodes with $1.5 \mathrm{~mm}$ touch proof connector (DIN 42 802) [21].

\subsubsection{Configuration and Control}

The configuration and control module consists of a high performance, low power, 8-bit microcontroller from Atmel® (ATxmega128A4U). It receives the orders from the user and sets the configuration of the other modules. It also works as a buffer for the data, between the AFE and the communication modules. The microcontroller works at $32 \mathrm{MHz}$, and can perform simple processing of the data. The communication with the other modules is done through serial peripheral interfaces (SPI), whose baud rate can be modified in the firmware between $1 \mathrm{M}$ bits per second (bps) to $10 \mathrm{Mbps}$.

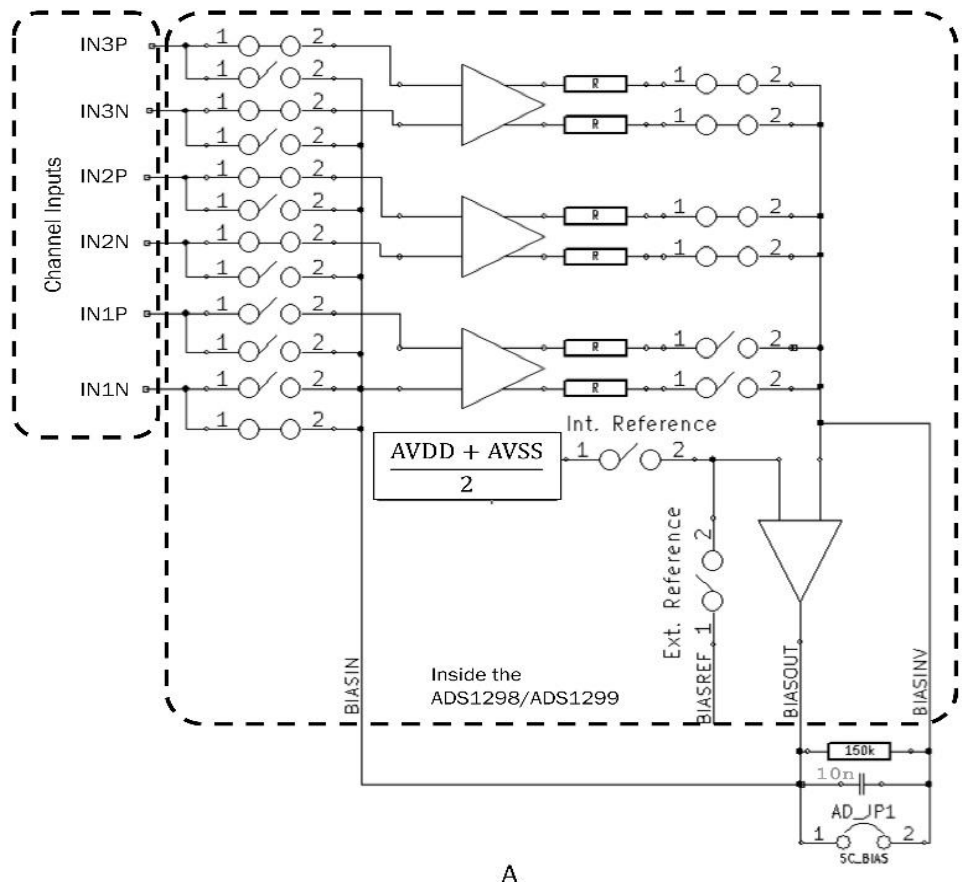

(a)

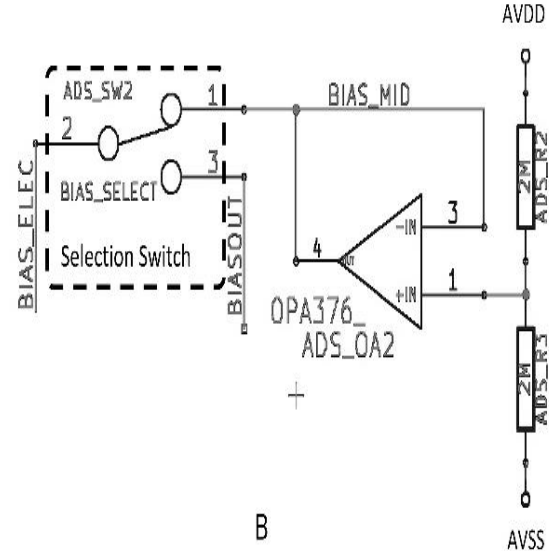

(b)

Figure 3. Bias signal circuit and internal diagram 
A. Internal diagram of the ADS1299 or ADS1298. Channels can be selected for the bias derivation in the GUI or firmware. In this figure, channels 2 and 3 generate the bias signal, which is then routed to the negative input of channel 1. B. User can choose the bias signal origin between the generated from the internal circuitry of the IC or the one at output of the operational amplifier which is the mid-supply voltage.

The general processes made by this module are shown in the flow diagram of Figure 4. For RFBrain and USB-Brain systems the microcontroller is in sleep mode at the beginning and all the other modules are off to save energy. After an "on-signal" is sent from the user, microcontroller wakes up and turns on all the other modules. The configuration parameters (like sampling frequency, gain and input multiplexer) are sent from the host PC. In the case of the Bluetooth-Brain system, the microcontroller is on and the Bluetooth transceiver is in discovery mode or connected to the receiver at the beginning. Settings must be configured in the microcontroller firmware by using a programmer device for the microcontroller and a software to load the code (for example the Atmel® Studio). Firmware of all systems was written in $\mathrm{C}$ and can be updated using the program and debug interface (PDI) port available in all the devices.

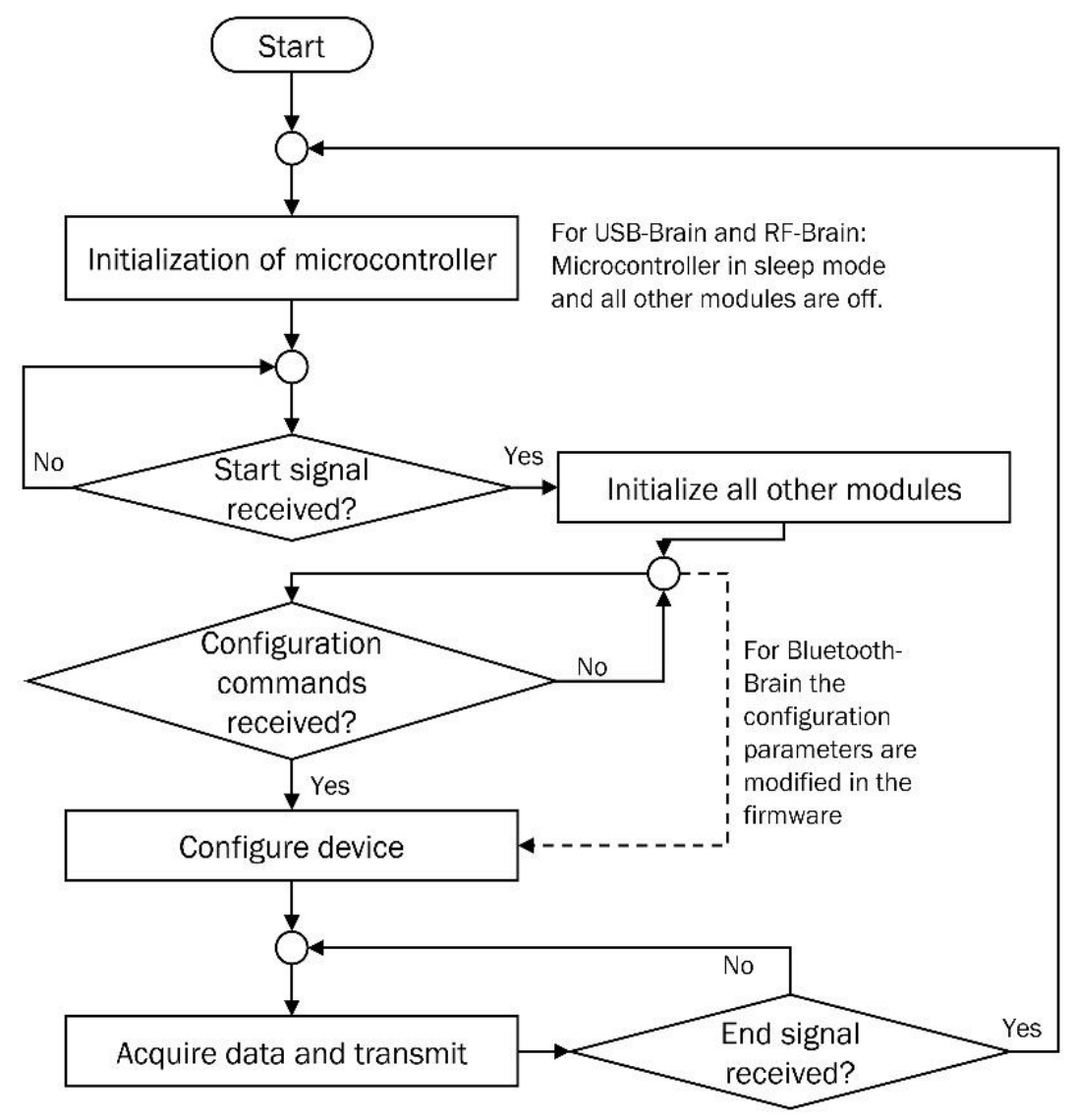

Figure 4. Flow diagram of the main processes in the configuration and control module

\subsection{RF-brain}

The first system of the family is formed by two boards: the first, a small and light transmitter is suitable for biofeedback and BCI applications, and is in charge of the acquisition, digitalization and transmission of data; the second, a USB receiver, is connected to a host computer for display, storage and processing of the signals. This device is an updated version of the Wireless Neuro Boards presented in [22], improving $60 \mathrm{~Hz} / 50 \mathrm{~Hz}$ noise isolation and providing more flexibility for the electrode configuration. Improvements were done by modifying all the connectors of the transmitter board and adding some components for the selection of reference and bias electrode, as explained in the previous section. 


\subsubsection{Transmitter}

The transmitter is a battery powered device formed by four modules: configuration and control, AFE, wireless RF communication and energy. For the communication, an RF transceiver from Nordic Semiconductor ${ }^{\circledR}$ was used (nRF24L01+). It operates at the industrial scientific and medical (ISM) band of $2.4 \mathrm{GHz}$ and is capable of reaching an on-air data rate of $2 \mathrm{Mbps}$, limiting the sampling frequency of the AFE which can be configured up to $2 \mathrm{k}$ samples per second (SPS), when all channels are active. If only one channel is active, the sampling frequency can be selected up to $4 \mathrm{kSPS}$.

The energy module generates a regulated $3.3 \mathrm{~V}$ required for all the other components in the board, from a standard $9 \mathrm{~V}$ battery. The maximum current consumption was estimated as $30.21 \mathrm{~mA}$ at a sampling frequency of 250 SPS, when all channels are active at programmable gain of 12; providing a battery life of more than 18 hours when using an alkaline battery of $550 \mathrm{mAh}$.

The device is a board approximately $60 \mathrm{~mm}$ long, $38 \mathrm{~mm}$ wide and $10 \mathrm{~mm}$ high. The weight of the transmitter is approximately $10 \mathrm{~g}$ without the $9 \mathrm{~V}$ alkaline battery whose weight is around $45 \mathrm{~g}$. Its weight makes this device appropriate for humans and animals of $500 \mathrm{~g}$ or more.

\subsubsection{Receiver}

In this device, control and configuration and RF-Communication modules are formed by the same components used for the transmitter. The USB communication module has a FTDI® FT232H IC, which is a converter from serial/parallel to Hi-speed USB, allowing transfer of data at baud rates from $115.2 \mathrm{kbps}$ to $1.38 \mathrm{Mbps}$. The receiver is powered from the USB port of the host computer, with an energy module to offer regulated $3.3 \mathrm{~V}$ to all the other modules.

\subsection{Bluetooth-brain}

Based on the architecture of the RF-Brain system, this portable device was designed to easily connect with smartphones and tablets through a Bluetooth modem (BlueSMiRF Silver from SparkFun Electronics $\left.{ }^{\circledR}\right)$. This modem is capable of transmitting data at a serial stream of $115200 \mathrm{bps}$, permitting the system to record EEG signals at 250 SPS. As shown in figure 1, the other modules are similar to those in the RF-Brain. When using a $9 \mathrm{~V}$ alkaline battery of $550 \mathrm{mAh}$, the system may work continuously for approximately 7 hours ( 8 channels active with programmable gain of 12). It is required for the receiver device to have Bluetooth connectivity (Bluetooth v2.0 or higher). Some embedded devices need a Bluetooth dongle to achieve this connectivity. For storage, visualization and processing of data, a GUI for Android devices was specially designed. MATLAB ${ }^{\circledR}$ GUI was also designed for the cases where the receiver is a Windows ${ }^{\circledR}$ laptop or PC. Basic functions are available: selection of channels of acquisition, visualization of signals and storage options.

\subsection{USB-brain}

This system was designed for experimentation where high sampling frequency is required and offers more options for electrode configuration and external hardware synchronization. Communication is made directly through the USB port of the host PC using a FTDI® serial/parallel to USB converter. Since the data do not need to be wirelessly transmitted, the sampling frequency can be set from 250 SPS to 4 kSPS.

The processing module uses the ATxmega128A4U microcontroller from Atmel ${ }^{\circledR}$ and a $32 \mathrm{~KB}$ SRAM chip was integrated for loading of files (for example, stimulation sequences when used to acquire evoked potentials) into the system. 8 general purpose input/output pins are available and can be used for different tasks such as synchronization with external hardware (for example, stimuli delivery) or interactions with the subject during the tests.

USB-Brain is powered through the USB port of the host computer. The power module delivers three voltage levels from the $5 \mathrm{~V}$ of a standard USB port: $\pm 2.5 \mathrm{~V}$ for the AFE and other analog modules, and $3.3 \mathrm{~V}$ for all the digital components. It also provides an isolation of $3 \mathrm{kVDC}$ from a DC/DC converter (Texas Instruments ${ }^{\circledR}$ DCH010505D) to ensure the safety of the subject under test. Transfer of data is also isolated through a digital coupler (Texas Instruments ${ }^{\circledR}$ ISO150) to avoid any current flow from the PC or laptop to the subject.

Total current consumption of the system during normal operation is approximately $117 \mathrm{~mA}$ at a sampling frequency of 250 SPS and a channel programmable gain of 12, with all channels active in differential mode. The dimensions of the main board of the system are: $91 \mathrm{~mm}$ wide by $112 \mathrm{~mm}$ long by 10 $\mathrm{mm}$ height, and its weight is $100 \mathrm{~g}$.

\subsection{Configuration and Acquisition Interfaces}

GUIs were implemented in MATLAB ${ }^{\circledR}$ for all the systems in the family when using a PC or laptop. From the GUIs, the user can select the channel configuration, sampling frequency and the options of storage 
and visualization of the data. The options vary depending on the system. Figure 5 shows the GUI for the USB-Brain system. For the RF-Brain and USB-Brain, in the current GUIs, the following options are available:

a. Short Circuit: All inputs shorted. For the RF-Brain all inputs connected internally to the reference electrode. For the USB-Brain, the inputs are connected either positive input with negative input or positive input to reference electrode, depending on the electrode configuration. These settings provide a way to measure noise levels in the system.

b. Test Signal: When activated, an auto-generated square signal from the AFE, with amplitude of $1 \mathrm{mV}$ and a frequency of $1 \mathrm{~Hz}$ approximately, is generated and helps to verify the correct acquisition and communication link in the case of the RF-Brain.

c. Normal: The normal operation of the channels for EEG acquisition.

For Bluetooth-Brain, these options can be modified from the firmware of the system. Table 2 shows the available options for all the devices. Additionally, for the Bluetooth-Brain, an Android GUI was designed where the user can select channels to visualize the data and store the signals into a file for further off-line processing.

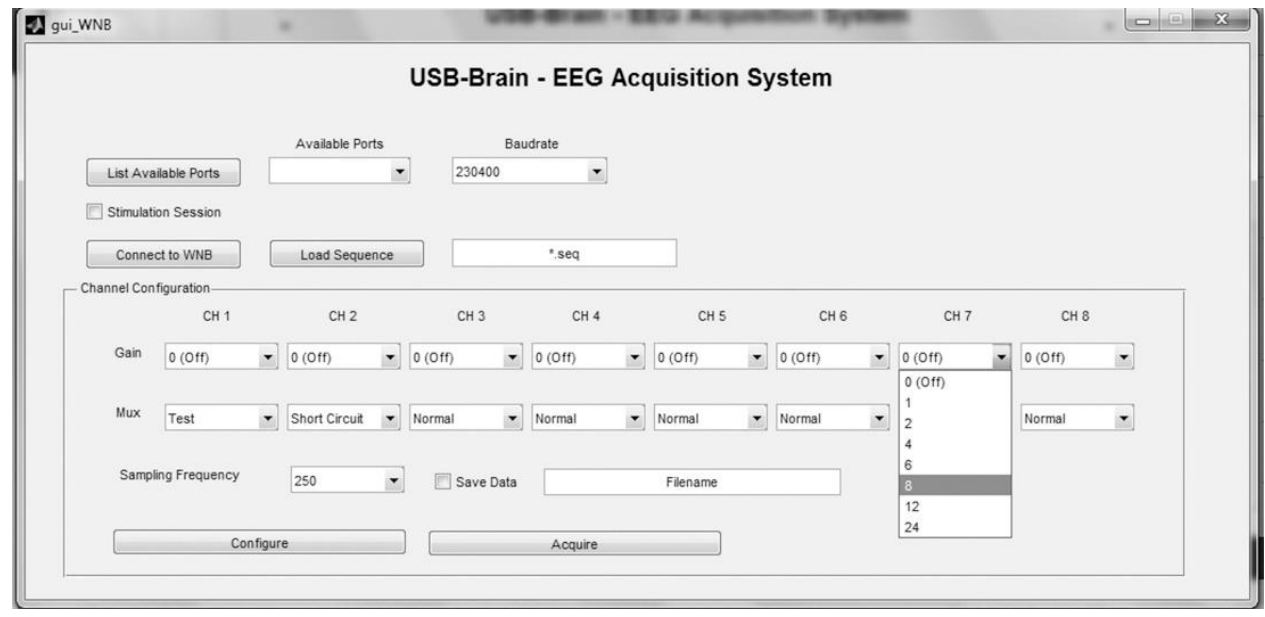

Figure 5. GUI for the USB-brain. user can choose the main features of the system to accomplish the requirements of the experiment

Table 2. Features of The Brain Family of Systems.

\begin{tabular}{|c|c|c|c|}
\hline Option & RF-Brain & Bluetooth-Brain $^{\mathrm{a}}$ & USB-Brain \\
\hline EEG channels & 8 & 8 & 8 \\
\hline Individual channel gain & Off, $1,2,3,4,6,8$ or 12 & Off, $1,2,3,4,6,8$ or 12 & Off, $1,2,4,6,8,12$ or 24 \\
\hline Sampling frequency & 8 channels: 250 SPS to $2 \mathrm{kSPS}$ & 8 channels: 250 SPS & 250 SPS to $4 \mathrm{kSPS}$ \\
\hline & 1 channel: 250 SPS to 4 kSPS & 1 channel: 250 SPS to $2 \mathrm{kSPS}$ & \\
\hline Reference & $\begin{array}{l}\text { Reference electrode or any } \\
\text { channel electrode }^{b}\end{array}$ & Bias/reference electrode & $\begin{array}{l}\text { In single-ended mode: } \\
\text { Reference electrode or any } \\
\text { channel electrode }^{\mathrm{b}}\end{array}$ \\
\hline Bias & $\begin{array}{l}\text { Bias electrode, any channel } \\
\text { electrode or internally } \\
\text { generated bias }\end{array}$ & Bias/reference electrode & $\begin{array}{l}\text { Bias electrode, any channel } \\
\text { electrode or internally } \\
\text { generated bias }^{c}\end{array}$ \\
\hline $\begin{array}{l}\text { Maximum power consumption } \\
\text { inactive }^{d}\end{array}$ & $2.64 \mathrm{~mA}$ & $66.7 \mathrm{~mA}$ & $77.7 \mathrm{~mA}$ \\
\hline $\begin{array}{l}\text { Data loss in transmission for a } \\
\text { range of } 5 \mathrm{~m}\end{array}$ & 0 packages & 0 packages & NA \\
\hline
\end{tabular}

${ }^{\mathrm{a}}$ For Bluetooth-Brain, the options can be selected by changing the firmware, not by the GUI.

${ }^{\mathrm{b}}$ External switch must be set according to the reference selection (see figure 2)

${ }^{\mathrm{c}}$ External switch must be set according to the bias selection (see figure 3 )

${ }^{\mathrm{d}}$ For RF-Brain and USB-Brain, microcontroller in sleep mode and all other modules off. For the Bluetooth-Brain, Bluetooth device in discovery mode.

${ }^{e}$ All channels active in normal operation, gain of 12 and sampling frequency of 250 SPS 


\subsection{Modularity}

All systems were designed to be modular with the purpose of making changes easily when the experimentation requires it. For this reason it was possible to make a whole family of systems by changing some modules in the design. Other possible modifications from the core of the family may include, for instance, the addition of more AFEs to increase the channel number, through a daisy-chain connection, avoiding the use of more pins in the microcontroller; for this modification the SPI communication baud rate and the sample rate must be taken into account: for a sample rate of $1 \mathrm{kSPS}$ and a SPI communication at 1 $\mathrm{MHz}, 5$ AFEs (with 8 channels each one) can be daisy-chained for a total number of 40 channels.

The communication module can be replaced in all systems, for example when other type of communication is required (wired or wireless). As long as an SPI communication protocol can be used, no major changes must be done in the firmware of the devices. When access to firmware is required, a PDI port is available in all the systems to change the firmware of the microcontroller and the basic configuration of the AFE.

\section{TESTS AND RESULTS}

All systems have different features; Table 2 summarizes the main characteristics of the family. The following aspects were tested for each system: power consumption, communication reliability (for the case of wireless devices) at all possible sampling rates, electric noise and acquisition of test signals. Pilot tests were done with human subjects to record EEG signals. During the tests the environment was not controlled, meaning that Wi-Fi signals and mobile cell phones were present in the area of tests.

\subsection{Power Consumption}

Power consumption was measured in all systems at standby and regular operation. The standby condition implies, for the RF-Brain and the USB-Brain, that the microcontroller is in sleep mode and all the other modules are off. For this condition, maximum power consumption of the devices were $2.64 \mathrm{~mA}$ and $77.7 \mathrm{~mA}$ for the RF-Brain and the USB-Brain respectively. Power module and extra components in the USBBrain system are responsible for its higher current consumption compared to the RF-Brain. In the case of the Bluetooth-Brain, the standby conditions means that the microcontroller is on and the Bluetooth transceiver is either in discovery mode or connected to the receiver device, but no data is transmitted. Its current consumption is $66.7 \mathrm{~mA}$.

In the case of regular operation, all channels in the systems were active in normal configuration, with a gain of 12 and a sampling frequency of 250SPS. For the RF-Brain, the maximum power consumption was $30.21 \mathrm{~mA}$; the Bluetooth-Brain has a maximum power consumption of $76.7 \mathrm{~mA}$ and the USB-Brain consumes at most $117.1 \mathrm{~mA}$.

\subsection{Communication Range and Reliability}

To test the communication range and reliability, a periodic saw-tooth signal was synthetically generated in the microcontroller and sent to the host computer or receiver for a minimum period of 10 minutes. All systems were tested at the complete set of the sampling frequencies. USB-Brain system communication did not present any data corruption or loss for all the sampling frequencies. Tests were also performed for the two wireless systems, changing the distance between the receiver and the transmitter. No data loss or corruption was presented for a distance of $5 \mathrm{~m}$ or less from the receiver.

\subsection{Noise Measurement}

A noise test took place for a set of sampling frequencies. The test consisted of shorting the inputs of the amplifiers through the input multiplexer settings and acquiring the signals. Noise measurements were made for 1000 continuous readings. Average results of all channels are detailed in Table 3 .

Table 3. Input Referred Noise Measurements for The Systems ${ }^{\mathrm{a}}$

\begin{tabular}{|c|c|c|c|c|c|c|}
\hline \multirow{2}{*}{$\begin{array}{l}\text { Sampling } \\
\text { Frequency }\end{array}$} & \multicolumn{2}{|c|}{ RF-Brain } & \multicolumn{2}{|c|}{ Bluetooth-Brain } & \multicolumn{2}{|c|}{ USB-Brain } \\
\hline & $\mu \mathrm{Vrms}$ & $\mu \mathrm{Vpp}$ & $\mu \mathrm{Vrms}$ & $\mu \mathrm{Vpp}$ & $\mu \mathrm{Vrms}$ & $\mu \mathrm{Vpp}$ \\
\hline 250 SPS & 0.49 & 2.20 & 0.50 & 4.16 & 0.16 & 1.13 \\
\hline $500 \mathrm{SPS}$ & 0.52 & 3.25 & NA & NA & 0.23 & 1.61 \\
\hline $1 \mathrm{kSPS}$ & 0.72 & 4.44 & NA & NA & 0.32 & 2.26 \\
\hline $2 \mathrm{kSPS}$ & 0.91 & 6.56 & NA & NA & 0.46 & 3.20 \\
\hline $4 \mathrm{kSPS}$ & NA & NA & NA & NA & 0.65 & 4.53 \\
\hline
\end{tabular}

${ }^{\mathrm{a}}$ Gain was set at 12 for all systems. 


\subsection{Test Signal}

To assess the integrity of the PGAs, its adequate calibration and visualization capability of the graphic interface, a test signal was acquired through all the channels of the three systems, at different gain settings. The test signal is an internally generated (generated inside the AFE ICs), square wave of $1 \mathrm{mV}$ of amplitude and $1 \mathrm{~Hz}$ of frequency. An example of the acquired test signal is shown in Figure 6. Test signal acquired with the bluetooth-brain. Test signal is internally generated by the IC in the AFE module and consists on a squared wave of $1 \mathrm{mV}$ of amplitude and $1 \mathrm{~Hz}$ of frequency

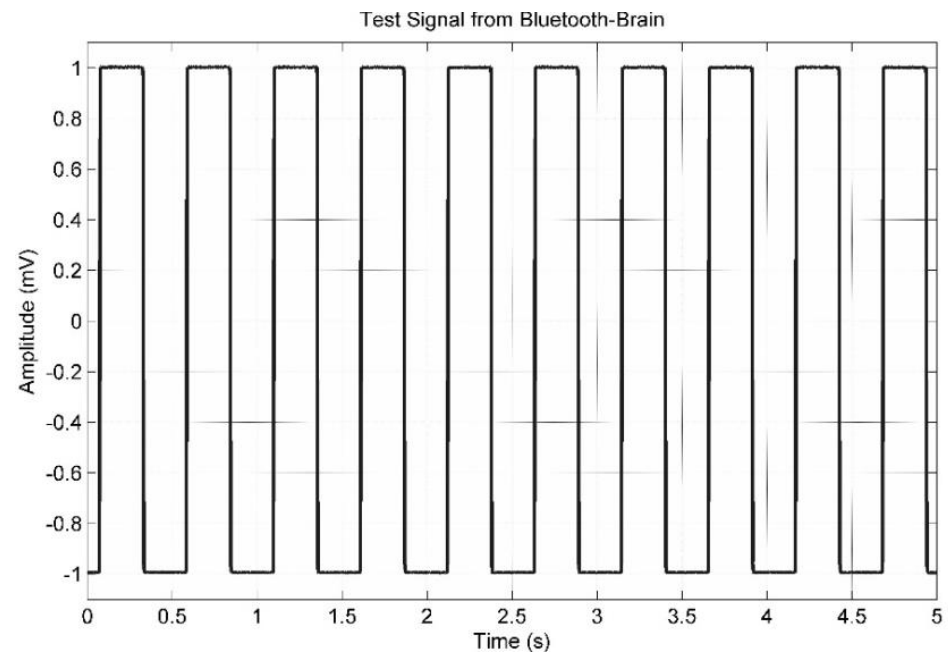

Figure 6. Test signal acquired with the bluetooth-brain

\subsection{Pilot Tests}

To evaluate the quality of the acquired physiological signals, a variety of tests were made for each system. All physiological signals were acquired from healthy human subjects who gave consent for the use of the data. Electrode location follows the standard ten-twenty electrode system [23], [24]. All procedures explained in this section were approved by the Research Ethical Committee of Universidad de los Andes-Bogotá-Colombia, or the Institutional Review Board of the University of Miami-Florida-United States, in fulfillment of the Code of Ethics of the World Medical Association. The following tests were done:

RF-Brain: auditory evoked potentials (AEPs) were acquired with the RF-Brain system from one subject (S1, 27 years old) using a data rate of 500 SPS and a gain of 12 in the PGAs. The experimental setup consisted on an electrode located in $\mathrm{Cz}$ and referred to $\mathrm{A} 1$ for ipsilateral acquisition. Bias signal was selected at mid-supply voltage and located also at A1. The stimulus used was monaural rarefaction clicks at $60 \mathrm{~dB}$ $\mathrm{nHL}$ at a frequency of 6 stimuli per second. Etymotic Research ER3 insert earphones were used. We wanted to assess the performance of the RF-Brain by comparing the signals acquired against signals from a commercial system; for this purpose we used the Intelligent Hearing Systems (IHS) Universal Smart Box at a sampling frequency of 500 SPS and a gain of 100000 (high-pass filter at $1 \mathrm{~Hz}$ and low-pass filter at $1500 \mathrm{~Hz}$ ). A total of 720 sweeps were recorded and averaged to extract the evoked potential. Figure 7 shows the results for the RF-Brain and the commercial system. The typical shape of an AEP (middle latency response) can be observed: two positive waves (marked as $\mathrm{Pa}$ and $\mathrm{Pb}$ ), and two negative waves $(\mathrm{Na}$ and $\mathrm{Nb}$ ) [25]. A difference in the amplitude of the signals is observed and may be due to the use of filters in the IHS system. Latencies and morphology of the signals are similar, taking into account the number of sweeps used for the computation of the potentials (see [3]).

Additionally, EEG signals were registered from another subject (S2, male 27 years old), with one electrode located at $\mathrm{Oz}$ and referred to Fpz. Sampling rates of 500 SPS and $1 \mathrm{kSPS}$ were used at a gain of 12. Alpha waves were observed for the periods of time where the subject had his eyes closed [21].

Bluetooth-Brain and USB-Brain: Following a similar procedure than for the RF-Brain, EEG signals were acquired with the Bluetooth-Brain and the USB-Brain, from two human subjects (S3, male, 22 years old and S4, male, 29 years old) using an electrode in Oz referred to Fpz, for a sampling rate of 250 SPS and a gain of 12 in the case of the Bluetooth-Brain. For the USB-Brain, sampling frequencies of 2 kSPS and 4 kSPS were used for a gain of 24 in the PGAs. An example of these acquired signals is shown in Figure 8, 
where a time-frequency representation is also depicted to view main frequency components. Alpha waves can be observed when the subject closes his eyes at approximately $10 \mathrm{~Hz}$.

Figure 7 shows auditory evoked potentials acquired with RF-brain system and the IHS universal smart box. 720 sweeps were averaged to obtain the evoked potentials. main waves of a typical AEP are observed and marked as "Na", "Pa", "Nb" and "Pb". A small difference in the amplitude can be observed, but latencies and morphology of the signals are similar. Figure 8 shows EEG signal acquired with the bluetooth-brain for subject S3 along with its time-frequency spectrogram to show its main frequency components. Electrodes were located in $\mathrm{Oz}$ with reference and bias in Fpz. alpha waves (at $10 \mathrm{~Hz}$ approximately) can be observed after the subject closes his eyes

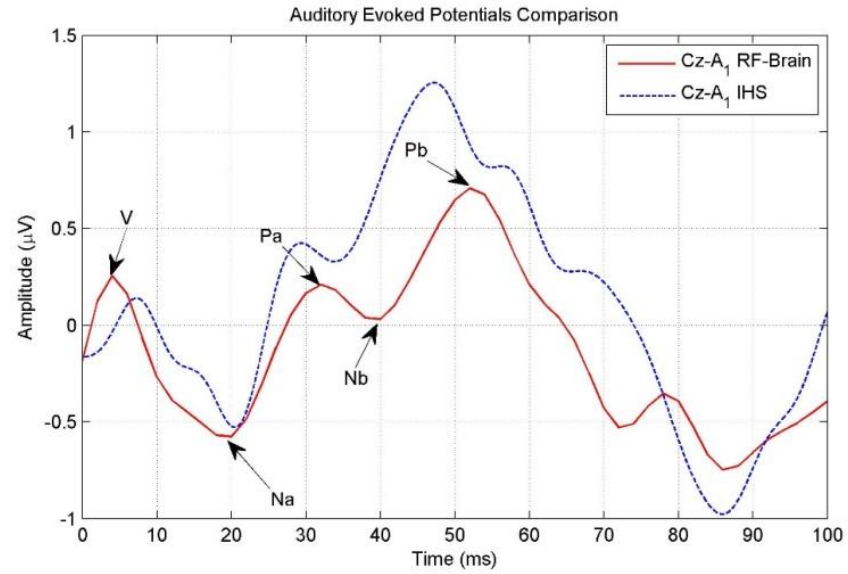

Figure 7. Auditory evoked potentials acquired with RF-brain system and the IHS universal smart box
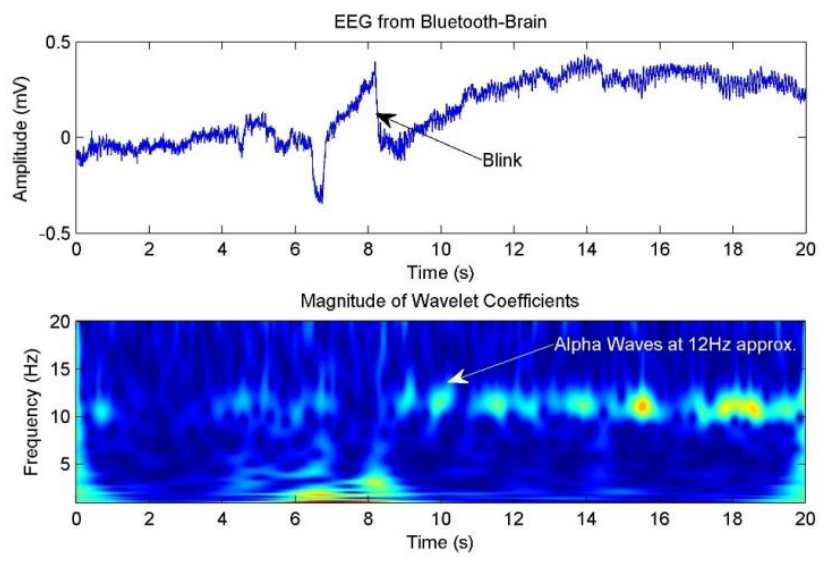

Figure 8. EEG signal acquired with the bluetooth-brain for subject S3 along with its time-frequency spectrogram to show its main frequency components

\section{DISCUSSION}

Three modular, flexible, affordable and high-performance systems were described in detail in this paper. All members of the family of systems have a common core composed by an AFE and a control and configuration module. Two wireless systems were designed for BCI and biofeedback applications where movement of the subjects is not restricted: RF-Brain and Bluetooth-Brain. Since these two systems are battery powered instead of being connected to the line power, the safety of the subject under test is assured. Bluetooth-Brain offers the possibility of connecting the system to a mobile device like a tablet or smartphone. The third, a wired system, offers options for experimentation where high sampling rates, synchronization and general purpose ports are needed. Flexibility is the result of the modular and open nature of the devices, which allows the selection of main features through GUIs and firmware upgrades for the control and configuration module.Access to all systems information, including schematics and $\mathrm{C}$ code of the microcontroller can be provided after contacting the authors.

A variety of tests were made to assess the performance of the family, including power consumption, range and integrity of communication link, synthetic signals and a pilot test with electrophysiological signals. Results have been positive, showing a performance similar or better to other systems like the ones presented in [26]-[29]. A comparison between the Brain family and other systems is shown in Table 4.

Although some commercial systems, like Cognionics 32-channel headset, have a greater number of channels [27], brain family has a higher sampling frequency and the same bit resolution. In fact, compared with any other system listed in table 4, brain family devices may be set at a higher sampling frequency. Another aspect to mention is their autonomy: while other systems are capable of working continuously for up to 5 hours, brain family systems can work for more than 18 hours with a standard $9 \mathrm{~V}$ alkaline battery.

Costs of manufacturing the systems are lower than the price of devices like the Emotiv Epoc (https://emotiv.com/epoc.php) or the ABM B-Alert X10 (http://www.advancedbrainmonitoring.com/), accomplishing one of the main goals of the implementation of the brain family, which was to offer high performance devices at a lower price.

One factor to be accounted for the brain family systems is the electrode positioning, which must be done manually with wet electrodes. This may require more time at the beginning of experimentation than 
when using other devices for which the electrodes are located in a structure with pre-defined locations. However, some locations or structures of electrodes are not suitable for all head sizes and for the cases where dry electrodes are used, the pressure required for the fixation of the sensors generates discomfort in the subjects [13]. This is avoided with the gel electrodes. Also, the lack of a pre-defined structure allows the adaptation for any head size or the use of non-standard locations of electrodes.

Table 4. Comparison of the Systems Against Other Similar Devices.

\begin{tabular}{|c|c|c|c|c|c|c|}
\hline \multirow[b]{2}{*}{ Feature } & \multicolumn{6}{|c|}{ Value } \\
\hline & $\begin{array}{c}\text { Brain Family } \\
\text { (RF/Bluetooth/USB) }\end{array}$ & Brown et al. [26] & Chi et al. [27] & $\begin{array}{c}\text { Lin CT et al [28] } \\
\text { Mindo-4 }\end{array}$ & $\begin{array}{l}\text { Emotiv } \\
\text { EPOC }^{\text {a }}\end{array}$ & $\begin{array}{l}\text { ABM B- } \\
\text { Alert X10 }\end{array}$ \\
\hline Channels & 8 & 8 & 32 & 4 & 14 & 9 \\
\hline Type of electrode & Passive & Active & Active & Passive & Passive & Passive \\
\hline $\begin{array}{l}\text { Electrode } \\
\text { connection }\end{array}$ & Gel & Direct & Direct & Direct & $\begin{array}{c}\text { Saline- } \\
\text { infused felt }\end{array}$ & $\begin{array}{l}\text { Gel-infused } \\
\text { foam }\end{array}$ \\
\hline $\begin{array}{l}\text { Sampling } \\
\text { frequencies } \\
\text { (SPS) }\end{array}$ & 250 to $4000^{\mathrm{c}}$ & 500 or 1000 & 250 & 128 to 512 & 128 & 256 \\
\hline $\begin{array}{l}\text { ADC resolution } \\
\text { (bits) }\end{array}$ & 24 & 11 & 24 & 24 & 14 & 16 \\
\hline Transfer & RF / Bluetooth / USB & RF & Bluetooth & RF & $\mathrm{RF}$ & Bluetooth \\
\hline $\begin{array}{l}\text { Supply voltage } \\
\text { (V) }\end{array}$ & $9 / 9 / 5$ & - & - & - & - & 3.7 \\
\hline $\begin{array}{l}\text { Supply current } \\
(\mathrm{mA})\end{array}$ & $30.21 / 76.7 / 117.1$ & 4.3 approx. & - & - & - & 40 approx. \\
\hline $\begin{array}{l}\text { Battery life } \\
\text { (hours) }\end{array}$ & $18 / 7 /-$ & 30 & 5 approx. & 23 & $>12$ & $>8$ \\
\hline $\begin{array}{l}\text { Input referred } \\
\text { noise }(\mu \text { Vrms })\end{array}$ & $0.49^{\mathrm{d}} / 0.50^{\mathrm{d}} / 0.16^{\mathrm{d}}$ & - & 1 & - & - & 2 approx. \\
\hline CMRR (dB) & $115 / 115 / 110$ & - & 100 & - & 85 approx. & 105 \\
\hline Price (USD) & $450^{\mathrm{e}}$ & - & - & - & $799^{\mathrm{f}}$ & 9950 \\
\hline $\begin{array}{l}{ }^{\mathrm{a}} \text { System from Em } \\
{ }^{\mathrm{b}} \text { System from Ad } \\
\text { http://www.advan } \\
{ }^{\mathrm{c}} \text { Depending on th } \\
{ }^{\mathrm{d}} \text { For normal opera } \\
{ }^{\mathrm{e}} \text { Rough price per } \\
{ }^{\mathrm{f}} \text { Price of the prod }\end{array}$ & $\begin{array}{l}\text { iv Inc. Information retri } \\
\text { nced Brain Monitoring } \\
\text { dbrainmonitoring.com/ } \\
\text { specific system and the } \\
\text { on, } 8 \text { channels on, gain } \\
\text { stem including electroni } \\
\text { t including access to raw }\end{array}$ & $\begin{array}{l}\text { ed from the manufa } \\
\text { Information retrie } \\
\text { mber of acquisition } \\
12 \text { and sampling fre } \\
\text { omponents and PC } \\
\text { EG signal }\end{array}$ & $\begin{array}{l}\text { r website: https } \\
\text { from the manuf: } \\
\text { nels (see Table } \\
\text { icy of } 250 \text { SPS. } \\
\text { anufacturing. }\end{array}$ & $\begin{array}{l}\text { otiv.com/epoc.php } \\
\text { er website: }\end{array}$ & & \\
\hline
\end{tabular}

\section{CONCLUSION}

A group of affordable and flexible EEG acquisition systems was described in detail in this paper. To assess the performance of the devices, a variety of tests took place. The results for all systems with synthetic test signals and electrophysiological signals showed that the devices are suitable tools for research areas involving EEG signals.

The cost of the devices (less than 500 USD each one) marks an important advantage over commercial systems. However, the manufacturing process may be time consuming and require a thorough knowledge of electronics. For the wireless devices, further improvements in the systems may include the use of an internal and rechargeable battery, even though the use of an external, alkaline battery provides enough autonomy for most of the experiments.

Modularity of the systems enables the researcher to independently manage the acquisition, processing and transmission of the signals to a host computer. This fragmentation allows the researcher to make decisions about the settings required, in accordance with the restrictions imposed by the experimentation protocol in relation to the mobility of the subject, sampling frequency, gain and configuration of the electrodes. As a future project, with further modifications to the core of the devices, the authors want to provide a main board to which daughter boards can be attached, depending on the specific requirements of the experimentation in communication mode (wireless or wired), availability of synchronization ports, general purpose inputs or outputs and memory modules, among others.

\section{REFERENCES}

[1] B.J. Kim, M.H. Lee, and S.W. Lee, "Intention analysis based on brain signal for participation induction during rehabilitation", 2015, pp. 1-2.

[2] S. Lee, Y. Shin, S. Woo, K. Kim, and H.N. Lee, "Review of Wireless Brain-Computer Interface Systems", in Brain-Computer Interface Systems - Recent Progress and Future Prospects, R. Fazel-Rezai, Ed. InTech, 2013. 
[3] J.T. Valderrama, A. de la Torre, I. Alvarez, J.C. Segura, M. Sainz, and J.L. Vargas, "A flexible and inexpensive high-performance auditory evoked response recording system appropriate for research purposes", Biomed. Eng. Biomed. Tech., vol. 59, no. 5, Jan. 2014.

[4] E. Yin, Z. Zhou, J. Jiang, Y. Yu, and D. Hu, “A Dynamically Optimized SSVEP Brain\&amp;\#x2013;Computer Interface (BCI) Speller”, IEEE Trans. Biomed. Eng., vol. 62, no. 6, pp. 1447-1456, Jun. 2015.

[5] T.S. Mel'nikova, S.I. Andrushkyavichus, and V.N. Krasnov, "Diurnal Dynamics of Reactivity in a Stress Test in Patients with Depression", Neurosci. Behav. Physiol., vol. 45, no. 4, pp. 398-403, May 2015.

[6] T. Meyer et al., "The role of frontal EEG asymmetry in post-traumatic stress disorder", Biol. Psychol., vol. 108, pp. 62-77, May 2015.

[7] S.F. Azab et al., "Childhood temporal lobe epilepsy: correlation between electroencephalography and magnetic resonance spectroscopy: a case-control study”, Ital. J. Pediatr., vol. 41, no. 1, Dec. 2015.

[8] I. Mporas, V. Tsirka, E.I. Zacharaki, M. Koutroumanidis, M. Richardson, and V. Megalooikonomou, "Seizure detection using EEG and ECG signals for computer-based monitoring, analysis and management of epileptic patients", Expert Syst. Appl., vol. 42, no. 6, pp. 3227-3233, Apr. 2015.

[9] M. Valderrama et al., "Identifying an increased risk of epileptic seizures using a multi-feature EEG-ECG classification", Biomed. Signal Process. Control, vol. 7, no. 3, pp. 237-244, May 2012.

[10] I. Constant and N. Sabourdin, "Monitoring depth of anesthesia: from consciousness to nociception. A window on subcortical brain activity", Pediatr. Anesth., vol. 25, no. 1, pp. 73-82, Jan. 2015.

[11] Z. Liang et al., "EEG entropy measures in anesthesia", Front. Comput. Neurosci., vol. 9, Feb. 2015.

[12] M. Peker, B. Şen, and H. Gürüler, "Rapid Automated Classification of Anesthetic Depth Levels using GPU Based Parallelization of Neural Networks", J. Med. Syst., vol. 39, no. 2, Feb. 2015.

[13] W. David Hairston et al., "Usability of four commercially-oriented EEG systems", J. Neural Eng., vol. 11, no. 4, p. 046018, Aug. 2014.

[14] V. Mihajlovic, B. Grundlehner, R. Vullers, and J. Penders, "Wearable, Wireless EEG Solutions in Daily Life Applications: What are we Missing?", IEEE J. Biomed. Health Inform., vol. 19, no. 1, pp. 6-21, Jan. 2015.

[15] Y. Iwatani et al., "Ictal high-frequency oscillations on scalp EEG recordings in symptomatic West syndrome", Epilepsy Res., vol. 102, no. 1-2, pp. 60-70, Nov. 2012

[16] K. Kobayashi et al., "Detection of seizure-associated high-frequency oscillations above 500Hz", Epilepsy Res., vol. 88 , no. $2-3$, pp. $139-144$, Feb. 2010.

[17] S.H. Choi, M. Lee, Y. Wang, and B. Hong, "Estimation of Optimal Location of EEG Reference Electrode for Motor Imagery Based BCI Using fMRI", 2006, pp. 1193-1196.

[18] A.K.O. Paukkunen and R. Sepponen, "The effect of ground electrode on the sensitivity, symmetricity and technical feasibility of scalp EEG recordings", Med. Biol. Eng. Comput., vol. 46, no. 9, pp. 933-938, Sep. 2008.

[19] W. Speier, A. Deshpande, and N. Pouratian, "A method for optimizing EEG electrode number and configuration for signal acquisition in P300 speller systems", Clin. Neurophysiol., vol. 126, no. 6, pp. 1171-1177, Jun. 2015.

[20] A. Nonclercq and P. Mathys, "Quantification of Motion Artifact Rejection Due to Active Electrodes and DrivenRight-Leg Circuit in Spike Detection Algorithms", IEEE Trans. Biomed. Eng., vol. 57, no. 11, pp. 2746-2752, Nov. 2010.

[21] J.G. Webster and J.W. Clark, Eds., Medical instrumentation: application and design, 4th ed. Hoboken, NJ: John Wiley \& Sons, 2010.

[22] J.M. Lopez, J.C. Bohorquez, J. Bohorquez, M.A. Valderrama, and F. Segura-Quijano, "Wireless Electroencephalogram Acquisition System for Recordings in Small Animal Models", 2013, pp. 3-4.

[23] G.H. Klem, H.O. Lüders, H.H. Jasper, and C. Elger, "The ten-twenty electrode system of the International Federation. The International Federation of Clinical Neurophysiology", Electroencephalogr. Clin. Neurophysiol. Suppl., vol. 52, pp. 3-6, 1999.

[24] R.T. Pivik, R.J. Broughton, R. Coppola, R. J. Davidson, N. Fox, and M. R. Nuwer, "Guidelines for the recording and quantitative analysis of electroencephalographic activity in research contexts", Psychophysiology, vol. 30, no. 6, pp. 547-558, Nov. 1993.

[25] C. Borgmann, B. Roß, R. Draganova, and C. Pantev, "Human auditory middle latency responses: influence of stimulus type and intensity", Hear. Res., vol. 158, no. 1-2, pp. 57-64, Aug. 2001.

[26] L. Brown, J. van de Molengraft, R.F. Yazicioglu, T. Torfs, J. Penders, and C. Van Hoof, "A low-power, wireless, 8-channel EEG monitoring headset", 2010, pp. 4197-4200.

[27] Y.M. Chi, Y. Wang, Y.T. Wang, T.P. Jung, T. Kerth, and Y. Cao, "A Practical Mobile Dry EEG System for Human Computer Interfaces”, in Foundations of Augmented Cognition, vol. 8027, D. D. Schmorrow and C. M. Fidopiastis, Eds. Berlin, Heidelberg: Springer Berlin Heidelberg, 2013, pp. 649-655.

[28] Chin-Teng Lin, Chun-Hsiang Chuang, Chih-Sheng Huang, Yen-Hsuan Chen, and Li-Wei Ko, "Real-time assessment of vigilance level using an innovative Mindo4 wireless EEG system", 2013, pp. 1528-1531.

[29] Chin-Teng Lin et al., "Wireless and Wearable EEG System for Evaluating Driver Vigilance", IEEE Trans. Biomed. Circuits Syst., vol. 8, no. 2, pp. 165-176, Apr. 2014. 